\title{
Quantifying finite-momentum effects in the quark quasidistribution functions of mesons
}

\author{
T. J. Hobbs ${ }^{1,2, *}$ \\ ${ }^{1}$ Department of Physics, University of Washington, Seattle, Washington 98195, USA \\ ${ }^{2}$ Department of Physics, Southern Methodist University, Dallas, Texas 75205, USA
}

(Received 24 August 2017; published 23 March 2018)

\begin{abstract}
The recently proposed large momentum effective theory (LaMET) of Ji [1] has led to a burst of activity among lattice practitioners to perform and control the first pioneering calculations of the quasi-PDFs of the nucleon. These calculations represent approximations to the standard, exact PDFs defined as correlation functions of fields with lightlike separation, being instead correlations along a longitudinal direction of the operator $\gamma^{z}$; as such, they differ from standard PDFs by a perturbative matching condition with powersuppressed $1 / p_{z}^{2}$ corrections, becoming exact in the limit $p_{z} \rightarrow \infty$. Investigating the systematics of this behavior thus becomes crucial to understanding the validity of LaMET calculations. While this has been done using models for the nucleon, an analogous dedicated study has not been carried out for the $\pi$ and $\rho$ valence quark distribution functions. Using a constituent quark model, a systematic calculation is performed to estimate the size and $x$ dependence of the finite- $p_{z}$ effects in these quasi-PDFs, finding them to be potentially tamer for lighter mesons than for the collinear quasi-PDFs of the nucleon.
\end{abstract}

DOI: $10.1103 /$ PhysRevD.97.054028

\section{INTRODUCTION}

a. Motivation. The pion occupies a special position in the spectrum of light hadrons given its dual identities as the Goldstone boson associated with the spontaneous breaking of chiral symmetry in QCD as well as the lightest meson with nontrivial quark content. The fact that the pion is responsible for important contributions to the structure of the nucleon has been known for some time, and the recognition of this fact has led to the introduction of pionic modes into many models [2] of proton structure. At the same time, ambiguities remain concerning the structure of the pion itself (as well as of other light mesons) and its role in the nucleon's quark substructure and flavor asymmetries [3]. This fact has inspired a number of experimental measurements of the pion structure function or parton distribution functions (PDFs) over the years, including an approved JLab measurement of the $\pi$ structure function via spectator tagging in the Sullivan process [4]. Theoretical studies of the $\pi$ structure function have an extensive history, with many model calculations of the $\pi$ structure function making up an extensive literature [5-9] on the topic.

\footnotetext{
tjhobbs@smu.edu
}

Published by the American Physical Society under the terms of the Creative Commons Attribution 4.0 International license. Further distribution of this work must maintain attribution to the author(s) and the published article's title, journal citation, and DOI. Funded by SCOAP.
Parallel to these efforts, the recently proposed largemomentum effective theory (LaMET) of Ji [1] has raised the prospect of computing quasi-PDFs, which represent correlation functions of a spacelike operator $\gamma^{z}$ rather than the usual lightlike $\gamma^{+}$that occurs in the formal definition of the exact PDF, i.e., the quark density matrix element defined along a hypersurface tangential to the lightcone [10] - a fact which makes the latter undefinable on a Euclidean lattice as would be required for lattice gauge calculations. These objects have thus enjoyed a rapidly burgeoning phenomenology [11-14] in the past few years. Crucially for the quasi-PDFs, as the longitudinal momentum $p_{z}$ of the parent hadron is boosted away from the rest frame, the longitudinal separation over which quark fields are correlated is mapped onto the light front asymptotically. Effectively, convolutional and subleading $p_{z}$ dependent corrections to the quasi-PDF are suppressed for $p_{z} \rightarrow \infty$ such that, in the limit of large hadronic momentum, LaMET provides a means of computing the PDF with a precision limited only by the $p_{z}$-scale attainable in lattice calculations. In practice, however, efforts on the lattice up to the present time $[15,16]$ have been confined to relatively small $p_{z} \sim 1 \mathrm{GeV}$; this fact necessitates careful study of the size and behavior of such corrections to the quasi-PDF due to the finite size of $p_{z}$ in likely lattice calculations. While studies of this sort have been carried out for the nucleon using quark-diquark models [17], it is the goal of the present work to use a constituent quark model to analyze the potential finite- $p_{z}$ effects in the valence-region quasiPDF of light mesons, specifically, the $\pi$ and $\rho$ meson. 
Some analyses [18-20] of $\pi$ structure bearing on LaMET methods have recently emerged, but these have been in the context of different model frameworks and formalisms, with the preponderance of the focus up to the present time on the parton distribution amplitude (PDA), which is now being computed in the first lattice results [21]. Here, however, I undertake a concentrated analysis of the $\pi$ and $\rho$ valence quasi-PDFs for the purpose of assessing the ability of LaMET methodology to extract the high $x$ behavior of the meson valence PDFs $q_{\pi}(x)$ and $q_{\rho}(x)$. It should be pointed out that the comparative instability of the $\rho$ meson against decay makes lattice calculations for this state more challenging at present; it is included in this analysis to demonstrate the potential for extension to other light hadrons.

The remainder of this paper is as follows: Sec. II below describes in detail the theoretical framework-the method for computing the exact LF distributions of the $\pi$ and $\rho$ mesons as well as the corresponding calculations using the LaMET method. In Sec. III, I describe my method for determining the model parameters that enter the exact LF calculation as well as the size and $x$ dependence of the finite- $p_{z}$ corrections to the meson quasi-PDFs at several assumed values of $p_{z}$. A brief conclusion follows in Sec. IV.

\section{FORMALISM}

The universal parton distribution function (PDF) for a quark carrying momentum $k$ in a hadron of momentum $p$ is given by Fourier transforms of hadronic matrix elements of parton-level correlation functions at a renormalization scale $\mu^{2}$, viz.

$$
q^{\mathrm{LF}}\left(x, \mu^{2}\right)=\int \frac{d \xi^{-}}{4 \pi} e^{-i \xi^{-} k^{+}}\left\langle p\left|\bar{\psi}\left(\xi^{-}\right) \gamma^{+} \mathcal{U}\left(\xi^{-}, 0\right) \psi(0)\right| p\right\rangle
$$

$$
\tilde{q}\left(x, \mu^{2}, p_{z}\right)=\int \frac{d \xi_{z}}{4 \pi} e^{-i \xi_{z} k_{z}}\left\langle p\left|\bar{\psi}\left(\xi_{z}\right) \gamma^{z} \mathcal{U}\left(\xi_{z}, 0\right) \psi(0)\right| p\right\rangle
$$

in which the quantities $\mathcal{U}(\xi, 0)$ above represent Wilson line insertions in the longitudinal direction either along the light-front or collinear to the $p_{z}$ boost, respectively, and the momentum fraction $x$ is defined according to $k^{+}=x p^{+}$in the exact PDF of Eq. (1) on the light front, or, analogously, along the direction of a longitudinal boost as $k_{z}=x p_{z}$ in the latter matrix element of Eq. (2) above. Following the work of $\mathrm{Ji}$ and others [1,22], this second quantity in Eq. (2), $\tilde{q}\left(x, \mu^{2}, p_{z}\right)$, is identified as the quasi-PDF, which receives both perturbative matching and subleading corrections in $1 / p_{z}^{2}$ that vanish in the limit $p_{z} \rightarrow \infty$. In fact, for nonsinglet quark densities such as are considered in this analysis of meson valence distributions there exists a matching condition of the form

$$
\begin{aligned}
\tilde{q}\left(x, \mu^{2}, p_{z}\right)= & \int d y Z\left(\frac{x}{y}, \frac{\Lambda}{p_{z}}, \frac{\mu}{p_{z}}\right) q^{\mathrm{LF}}\left(y, \mu^{2}\right) \\
& +\mathcal{O}\left(\frac{\Lambda^{2}}{p_{z}^{2}}, \frac{M^{2}}{p_{z}^{2}}\right),
\end{aligned}
$$

in which the convolution is with respect to a perturbative matching factor $Z$ that depends only upon the distributions' UV behavior with the parameter $\Lambda$ an associated regulator scale. As a consequence, at infinite hadronic momentum this LaMET framework renders the quasi-PDF $\tilde{q}\left(x, \mu^{2}, p_{z}\right)$ an asymptotically accurate approximation of the exact PDF $q\left(x, \mu^{2}\right)$ defined on the light front according to Eq. (1). I verify this point numerically in the context of a constituent quark model for both the $\pi$ and $\rho$ systems, with the goal of assessing the range and degree of validity of LaMET calculations for meson valence PDFs.

\section{A. Exact PDFs}

a. Pion LF distribution. For the purpose of this exploratory analysis, I use a simple pseudoscalar interaction for the $\pi$-quark coupling according to the Lagrangian

$$
\mathcal{L}_{\pi q s}=i N_{\pi}^{1 / 2} \bar{\psi}_{q} \gamma_{5} \varphi_{\pi} \psi_{s}+\text { H.c. }
$$

in which $\psi_{q, s}$ denote the intermediate quark fields depicted in Fig. 1(a) and $\varphi_{\pi}$ represents the pseudoscalar $\pi$ field. The underlying process depicted in Fig. 1 entails the short-lived dissociation of an initial-state meson of mass $M_{\pi, \rho}$ carrying momentum $p$ into an intermediate state involving its constituent quark degrees of freedom - an interacting quark of mass $m$ with momentum $k$ and a recoiling spectator quark of mass $m_{s}$ and momentum $q=p-k$ by conservation. Taking this as the basis for the meson model, for the exact $\mathrm{LF}$ valence quark distribution in the $\pi$, following the interaction defined by Eq. (4) and standard Feynman rules for Fig. 1(a), I write down the expression

$$
\begin{aligned}
q_{\pi}^{\mathrm{LF}}(x)= & \frac{N_{\pi}}{2(2 \pi)^{4}} \int d k^{+} d k^{-} d^{2} k_{\perp}\left(\frac{1}{2 p^{+}}\right) \delta\left(x-\frac{k^{+}}{p^{+}}\right) \\
& \times \operatorname{tr}\left(\gamma_{5}(\not k+m) \gamma^{+}(\not k+m) \gamma_{5}\left(-\not 1+m_{s}\right)\right) \\
& \times 2 \pi \delta\left(q^{2}-m_{s}^{2}\right)\left[\frac{\phi_{\pi}\left(k^{2}\right)}{\left(k^{2}-m^{2}\right)}\right]^{2},
\end{aligned}
$$

wherein I have used the usual definition $[23,24]$ of the forward cut of the struck quark line to isolate the leadingtwist (LT) contribution, i.e.,

$$
\text { LT quark-line cut } \rightarrow \frac{\gamma^{+}}{2 p^{+}} \delta\left(x-\frac{k^{+}}{p^{+}}\right) .
$$

The overall normalization $N_{\pi}$ of the $\pi$ valence quark distribution $q_{\pi}^{\mathrm{LF}}(x)$ is related to the $\pi$-quark interaction strength of Eq. (4) and is chosen so as to ensure the probability conservation condition is always satisfied: 

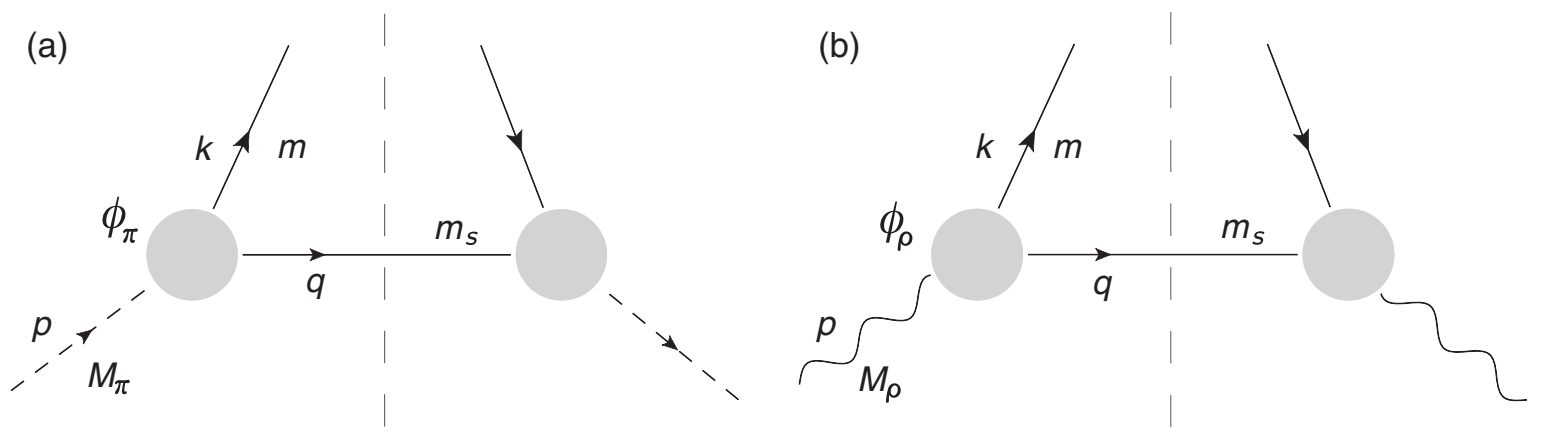

FIG. 1. (a) The diagram contributing to the leading-twist valence distribution of the pion in the relativistic constituent quark model following from the interactions defined in Eq. (4). (b) The corresponding graph for the spin-1 $\rho$ meson following the Pauli interaction of Eq. (12). In the model, the leading twist cut corresponds to a photon resolving a struck quark of momentum $k$ emerging from an initialstate meson with external momentum $p$ with a spectator quark carrying momentum $q=p-k$. The meson-quark vertex is regulated by an appropriate choice of phenomenological function $\phi_{M}\left(k^{2}\right)$ as defined in Eq. (8). Note that in general the values of the quarks' constituent masses $m$ and $m_{s}$ in panel (a) need not be identical to those in panel (b).

$$
\int_{0}^{1} d x q_{\pi}^{\mathrm{LF}}(x)=1
$$

The quark-meson interaction leading to Eq. (5) is inherently nonperturbative and in phenomenological models requires the introduction of a vertex factor to suppress ultraviolet divergences at large $k$. Here, I use a single-parameter covariant function of the struck quark's squared momentum,

$$
\phi_{M}\left(k^{2}\right) \equiv\left(\frac{\Lambda_{M}^{2}}{k^{2}-\Lambda_{M}^{2}}\right)^{3 / 4} \quad M=(\pi, \rho),
$$

for which I tune in Sec. III the values of the parameters $\Lambda_{M}$ to reproduce structure function moments for the $\pi$ and $\rho$ mesons that have been computed via lattice techniques $[25,26]$. While regulator functions with different dependence on $k^{2}$ are possible, I proceed with this analysis using the form in Eq. (8), given that this guarantees the nonlinear behavior $q_{v}(x) \sim(1-x)^{2}$ at $x \rightarrow 1$ expected on the basis of DysonSchwinger analyses [8], perturbative QCD arguments [27] and fits [28] to data on the $\pi$ structure function obtained via the Drell-Yan process [29]. Also, in general expressions like Eq. (8) above, quantities that depend upon the choice of initial state meson (i.e., either $\pi$ or $\rho$ ) are indexed with the subscript $M$ for compactness, and the qualitative results of this study do not depend on the parametric choice for $\phi_{M}\left(k^{2}\right)$.

Evaluating the expression in Eq. (5) according to standard techniques, integrating the $\delta$ function in $q^{2}-m_{s}^{2}$ over $k^{-}$and using the on-shellness of the struck quark on the light-front, I obtain the expression for the $\pi$ valence quark exact PDF,

$$
\begin{aligned}
q_{\pi}^{\mathrm{LF}}(x)= & \frac{N_{\pi}}{8 \pi^{2}} \int \frac{d k_{\perp}^{2}}{x^{2}(1-x)}\left\{k_{\perp}^{2}+\left(x m_{s}+(1-x) m\right)^{2}\right\} \\
& \times\left[\frac{\phi_{\pi}\left(t_{\pi}\right)}{\left(M_{\pi}^{2}-\hat{s}\right)}\right]^{2} \\
\hat{s}= & \frac{m^{2}+k_{\perp}^{2}}{x}+\frac{m_{s}^{2}+k_{\perp}^{2}}{1-x},
\end{aligned}
$$

where $\hat{s}$ denotes the partonic invariant mass of the internal 2-quark system and I note that, on the light-front, the covariant squared momentum of the struck quark after integrating $\int d k^{-} \delta\left(q^{2}-m_{s}^{2}\right)$ is given by

$$
\begin{aligned}
t_{M} & \equiv k_{M}^{2}=k^{+} k_{M}^{-}-k_{\perp}^{2} \\
& =\frac{1}{1-x}\left(-k_{\perp}^{2}-x m_{s}^{2}+x(1-x) M_{M}^{2}\right) .
\end{aligned}
$$

Arriving at Eqs. (9)-(10) requires integrating over $k^{+}$and $k^{-}$ by means of the $\delta$ functions resulting from the cut in Fig. 1; namely, these are

$$
\begin{aligned}
\delta\left(x-\frac{k^{+}}{p^{+}}\right) & =p^{+} \delta\left(x p^{+}-k^{+}\right) \\
\delta\left(q^{2}-m_{s}^{2}\right) & =\frac{1}{(1-x) p^{+}} \delta\left(p^{-}-k^{-}-\frac{m_{s}^{2}+k_{\perp}^{2}}{(1-x) p^{+}}\right),
\end{aligned}
$$

where I have assumed integration over the first line in writing the second.

b. Exact rho meson distribution. It is possible to carry out a similar calculation for the $\rho$ meson, for which I take a minimal Lagrangian of the form

$$
\mathcal{L}_{\rho q s}=N_{\rho}^{1 / 2} \bar{\psi}_{q} \gamma_{\mu} \psi_{s} \vartheta_{\rho}^{\mu}+\text { H.c. },
$$

in which the vector field of the spin-1 $\rho$ meson is now denoted by $\vartheta_{\rho}^{\mu}$. Using this form of the interaction and evaluating the corresponding diagram shown in Fig. 1(b) I get the exact model expression for the valence quark distribution of the $\rho$ meson,

$$
\begin{aligned}
q_{\rho}^{\mathrm{LF}}(x)= & \frac{N_{\rho}}{2(2 \pi)^{4}} \int d k^{+} d k^{-} d^{2} k_{\perp}\left(\frac{1}{2 p^{+}}\right) \delta\left(x-\frac{k^{+}}{p^{+}}\right) \\
& \times\left(g^{\mu \nu}-\frac{1}{M_{\rho}^{2}} p^{\mu} p^{\nu}\right) \operatorname{tr}\left(\gamma_{\mu}(\not k+m) \gamma^{+}(\not k+m)\right. \\
& \left.\times \gamma_{\nu}\left(-\not q+m_{s}\right)\right) 2 \pi \delta\left(q^{2}-m_{s}^{2}\right)\left[\frac{\phi_{\rho}\left(k^{2}\right)}{\left(k^{2}-m^{2}\right)}\right]^{2} .
\end{aligned}
$$



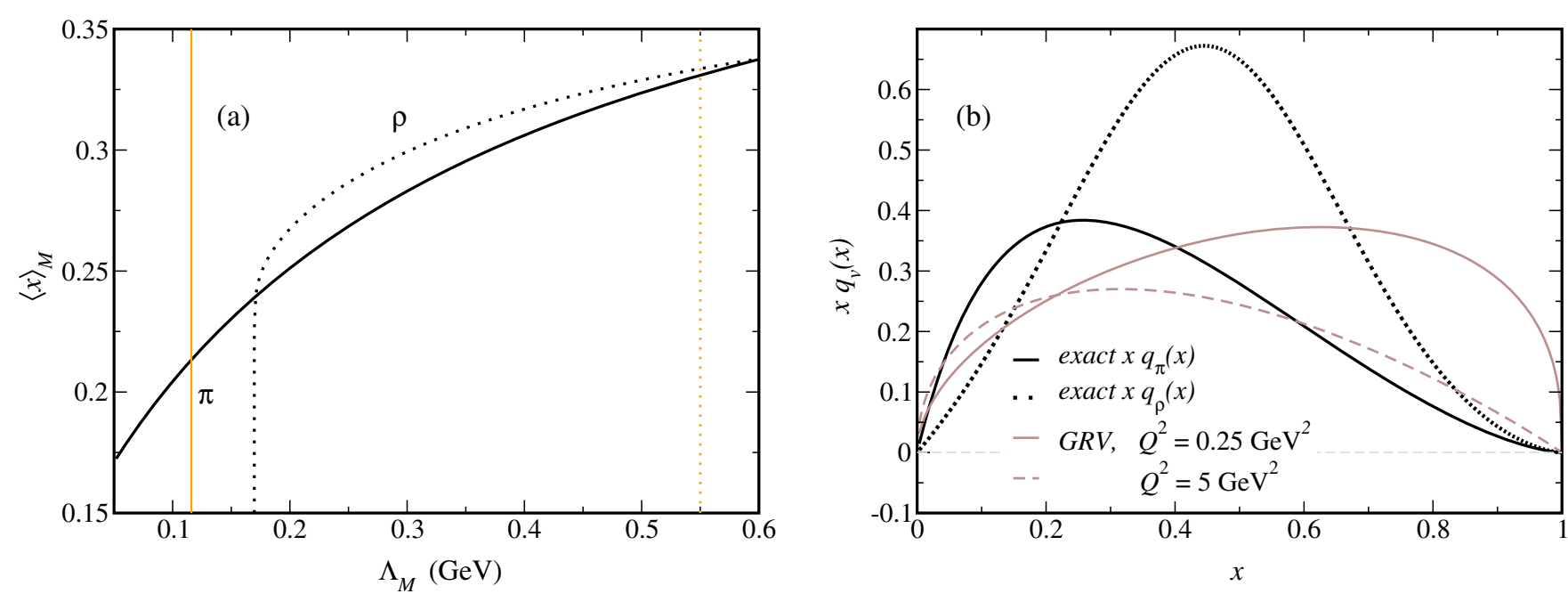

FIG. 2. (a) A plot of the dependence of the first moments of the mesons' exact LF valence distributions $\langle x\rangle_{M}$ of Eq. (24) as a function of the UV regulators $\Lambda_{M}$ appearing in Eq. (8) for both $M=\pi$ (solid curve) and $M=\rho$ (dotted curve). The associated vertical bars mark the specific values to which I tune $\Lambda_{M}$ so as to reproduce the lattice-computed moments for these distributions found in Ref. [25] for the $\pi$ (solid bar) and Ref. [26] for the $\rho$ (dotted bar). (b) The exact LF valence distributions $x q_{v}^{\mathrm{LF}}(x)$ corresponding to these choices for $\Lambda_{M}$. The line styles for the darker curves match those of panel (a), while the lighter brown curves are the output of the GRV $\pi$ distribution global analysis in Ref. [30] at two low scales of $Q^{2}$ as noted in the legend.

In this case, the higher spin of the $\rho$ generates a more convoluted momentum dependence from the trace algebra in the numerator of Eq. (13) above, ultimately leading to

$$
\begin{aligned}
q_{\rho}^{\mathrm{LF}}(x)= & \frac{N_{\rho}}{8 \pi^{2}} \int \frac{d k_{\perp}^{2}}{x^{2}(1-x)^{2}}\left\{k_{\perp}^{2}+4 x(1-x) m m_{s}\right. \\
& +\left(x m_{s}+(1-x) m\right)^{2}+\left(m^{2}+k_{\perp}^{2}+x^{2} M_{\rho}^{2}\right) \\
& \left.\times\left(\frac{m_{s}^{2}+k_{\perp}^{2}}{M_{\rho}^{2}}+(1-x)^{2}\right)\right\}\left[\frac{\phi_{\rho}\left(t_{\rho}\right)}{\left(M_{\rho}^{2}-\hat{s}\right)}\right]^{2} .
\end{aligned}
$$

I plot my numerical determination for this distribution alongside that of the $\pi$ in Fig. 2 and describe the associated numerical method in Sec. III after describing the corresponding LaMET calculation below.

\section{B. LaMET approach}

In the LaMET formalism the cut in the struck quark lines of Fig. 1 given by Eq. (6) for the exact calculation must be replaced by the modified leading-twist operator insertion

$$
\frac{\gamma^{+}}{2 p^{+}} \delta\left(x-\frac{k^{+}}{p^{+}}\right) \rightarrow \frac{\gamma^{z}}{2 p_{z}} \delta\left(x-\frac{k_{z}}{p_{z}}\right)
$$

such that in the present constituent quark model Eq. (5) becomes for the $\pi$ quasi-PDF

$$
\begin{aligned}
\tilde{q}_{\pi}\left(x, p_{z}\right)= & \frac{N_{\pi}}{(2 \pi)^{4}} \int d k^{0} d k_{z} d^{2} k_{\perp}\left(\frac{1}{2 p_{z}}\right) \delta\left(x-\frac{k_{z}}{p_{z}}\right) \\
& \times \operatorname{tr}\left(\gamma_{5}(\not k+m) \gamma^{z}(\not k+m) \gamma_{5}\left(-\not h+m_{s}\right)\right) \\
& \times 2 \pi \delta\left(q^{2}-m_{s}^{2}\right)\left[\frac{\phi_{\pi}\left(k^{2}\right)}{\left(k^{2}-m^{2}\right)}\right]^{2} .
\end{aligned}
$$

For the quasi-PDF calculation at finite $p_{z}$ I require explicit expressions in terms of $x$ and $k_{\perp}^{2}$ that result from integrating the $\delta$ function of Eq. (16), $\int d k^{0} \delta\left(q^{2}-m_{s}^{2}\right)$, according to the Cutkosky procedure; this again has the effect of rendering the kinematics as appropriate for an onshell spectator particle. As with the LF calculation before, doing so results in the analogue of Eq. (11), but for the LaMET quasidistributions; I use

$$
\begin{aligned}
& \delta\left(x-\frac{k_{z}}{p_{z}}\right)=p_{z} \delta\left(x p_{z}-k_{z}\right) \\
& \delta\left(q^{2}-m_{s}^{2}\right)=\frac{1}{2\left(p^{0}-k^{0}\right)} \delta\left(p^{0}-k^{0}-\sqrt{m_{s}^{2}+k_{\perp}^{2}+(1-x)^{2} p_{z}^{2}}\right) .
\end{aligned}
$$

I denote quantities subsequent to the integrations over $k^{0}$ and $k_{z}$ in Eq. (16) using Eq. (17) as $\tilde{p}_{M}$, etc. In particular, several scalar products are needed:

$$
\begin{aligned}
\tilde{p}_{M} \cdot \tilde{k}_{M} & =\tilde{p}_{M}^{0} \tilde{k}_{M}^{0}-x p_{z}^{2} \\
\tilde{p}_{M} \cdot \tilde{q} & =\tilde{p}_{M}^{0} \tilde{q}^{0}-(1-x) p_{z}^{2} \\
\tilde{q} \cdot \tilde{k}_{M} & =\tilde{q}^{0} \tilde{k}_{M}^{0}+k_{\perp}^{2}-x(1-x) p_{z}^{2},
\end{aligned}
$$


for which the relevant energies $\tilde{p}_{M}^{0}$, etc., are

$$
\begin{aligned}
\tilde{p}_{M}^{0} & \equiv p_{z} \mu_{M} \\
\tilde{q}^{0} & \equiv(1-x) p_{z} \mu_{s} \\
\tilde{k}_{M}^{0} & \equiv \sqrt{M_{M}^{2}+m_{s}^{2}+k_{\perp}^{2}+\left(1+[1-x]^{2}\right) p_{z}^{2}-2(1-x)\left(1-\mu_{M} \mu_{s}\right) p_{z}^{2}} \\
\mu_{M} & \equiv \sqrt{1+\frac{M_{M}^{2}}{p_{z}^{2}}} \quad \mu_{s} \equiv \sqrt{1+\frac{m_{s}^{2}+k_{\perp}^{2}}{(1-x)^{2} p_{z}^{2}}},
\end{aligned}
$$

and similarly,

$$
\begin{aligned}
\tilde{k}_{M}^{2} & \equiv\left(\tilde{k}_{M}^{0}\right)^{2}-k_{\perp}^{2}-x^{2} p_{z}^{2} \\
& =M_{M}^{2}+m_{s}^{2}+2(1-x)\left(1-\mu_{M} \mu_{s}\right) p_{z}^{2} .
\end{aligned}
$$

Evaluating the trace and integrating over $k^{0}$ and $k_{z}$ in Eq. (16) using the $\delta$-function expressions of Eq. (17) leads to the $\pi$ quasidistribution in terms of the quantities defined in Eqs. (18)-(20):

$$
\begin{aligned}
\tilde{q}_{\pi}\left(x, p_{z}\right)= & \frac{N_{\pi}}{4 \pi^{2}} \int \frac{d k_{\perp}^{2}}{2(1-x) \mu_{s}} \\
& \times\left\{2 x\left(m m_{s}+\left(\tilde{q} \cdot \tilde{k}_{\pi}\right)\right)+\left(m^{2}-\tilde{k}_{\pi}^{2}\right)(1-x)\right\} \\
& \times\left[\frac{\phi_{\pi}\left(\tilde{k}_{\pi}^{2}\right)}{\left(M_{\pi}^{2}+m_{s}^{2}-m^{2}+2(1-x)\left(1-\mu_{\pi} \mu_{s}\right)\right.}\right]^{2} ;
\end{aligned}
$$

this expression may then be evaluated numerically at the same values of $m, m_{s}, \Lambda_{\pi}$, and $N_{\pi}$ found for the exact LF calculation of Eq. (9) by integrating over $k_{\perp}^{2}$. Moreover, I examine $\tilde{q}_{\pi}\left(x, p_{z}\right)$ at finite values of $p_{z}$ in Sec. III below and sketch the extent to which the high $x$ valence region of $q_{\pi}(x)$ may be accessed with the LaMET method.

The same formalism can be used for the $\rho$ to obtain an analogous $p_{z}$ dependent expression for the valence quasiPDF. Using the LaMET treatment of the cut quark line in Eq. (15) and writing the appropriate expression akin to Eq. (13) I get

$$
\begin{aligned}
\tilde{q}_{\rho}\left(x, p_{z}\right)= & \frac{N_{\rho}}{(2 \pi)^{4}} \int d k^{0} d k_{z} d^{2} k_{\perp}\left(\frac{1}{2 p_{z}}\right) \delta\left(x-\frac{k_{z}}{p_{z}}\right) \\
& \times\left(g^{\mu \nu}-\frac{1}{M_{\rho}^{2}} p^{\mu} p^{\nu}\right) \operatorname{tr}\left(\gamma_{\mu}(\not k+m) \gamma^{z}(\not k+m) \gamma_{\nu}\right. \\
& \left.\times\left(-\not q+m_{s}\right)\right) 2 \pi \delta\left(q^{2}-m_{s}^{2}\right)\left[\frac{\phi_{\rho}\left(k^{2}\right)}{\left(k^{2}-m^{2}\right)}\right]^{2},
\end{aligned}
$$

which, like the $\pi$ quasidistribution, closely mirrors its exact LF analogue, aside from its equal-time form and space-like operator structure. Evaluating this last expression as before at the relevant finite- $p_{z}$ kinematics again yields the desired formula in terms of the inner products of Eqs. (18)-(20):

$$
\begin{aligned}
\tilde{q}_{\rho}\left(x, p_{z}\right)= & \frac{N_{\rho}}{4 \pi^{2}} \int \frac{d k_{\perp}^{2}}{(1-x) \mu_{s}} \\
& \times\left\{x\left(\frac{2}{M_{\rho}^{2}}\left(\tilde{p}_{\rho} \cdot \tilde{q}\right)\left(\tilde{p}_{\rho} \cdot \tilde{k}_{\rho}\right)+3 m m_{s}+\left(\tilde{q} \cdot \tilde{k}_{\rho}\right)\right)\right. \\
& \left.+\frac{1}{2}\left(m^{2}-\tilde{k}_{\rho}^{2}\right)\left(\frac{2\left(\tilde{p}_{\rho} \cdot \tilde{q}\right)}{M_{\rho}^{2}}+(1-x)\right)\right\} \\
& \times\left[\frac{\phi_{\rho}\left(\tilde{k}_{\rho}^{2}\right)}{\left(M_{\rho}^{2}+m_{s}^{2}-m^{2}+2(1-x)\left(1-\mu_{\rho} \mu_{s}\right)\right.}\right]^{2} .
\end{aligned}
$$

This latter expression can also be computed for plausible values in the model parameter space, and I consider the side-by-side comparison of the LaMET $v s$ exact LF calculations of $q_{\rho}(x)$ along with $q_{\pi}(x)$ in Sec. III below.

\section{NUMERICAL RESULTS}

In Sec. II, I used the light-front and LaMET formalisms to derive the (quasi-)PDFs for the $\pi$ and $\rho$ valence quark content; this was done under the auspices of a model that couples the mesons to their constituent quarks according to the minimal Lagrangians of Eqs. (4) and (12). With this formalism established, I proceed in the present Section to fix the adjustable parameters that enter the constituent quark model, facilitating an analysis of the size and $x$ dependence of the finite- $p_{z}$ corrections that enter Eq. (2) for both the $\pi$ and $\rho$ distributions. Taking this as the primary goal of the present analysis - to study the ranges of validity of LaMET approximations to the exact meson PDFs in a typical model-it is sufficient to make plausible, physically motivated selections for the model parameters (mainly, the constituent quark masses $m$ and $m_{s}$ that enter the meson distributions derived in Sec. II). With these fixed, it is then possible to tune the remaining degrees of freedom [the UV cutoffs $\Lambda_{M}$ in Eq. (8)] to reproduce information determined in lattice QCD calculations (the distribution moments), thereby completely determining the constituent quark model and providing a basis for comparing the LaMET and LF meson distributions in the valence region.

For both the $\pi$ and $\rho$ distributions, I fix hadronic masses to their pole values $M_{\pi}=0.139 \mathrm{GeV}$ and $M_{\rho}=0.77 \mathrm{GeV}$. In the case of the intermediate states, I take for the constituent masses of the $\pi$ valence quarks the static values 
$m=m_{s}=M / 3 \approx 0.33 \mathrm{GeV}$, where $M$ is the proton mass. For the heavier $\rho$ meson, however, this choice would introduce numerical instabilities due to the presence of the quark denominators in Eq. (13), which produce potentially singular overall factors of $\left[M_{\rho}-\hat{s}\right]^{-2}$ in the final expression for $q_{\rho}^{\mathrm{LF}}(x)$ in Eq. (14). Essentially, the model in this circumstance is not sufficiently confining to prevent the decay of the $\rho$ into its lighter-mass partonic constituents. A simple reparative scheme to address this issue is the effective mass prescription [31,32], which involves the selection of a larger mass for the bound constituent quark; this is on the logic that the nonperturbative interactions necessary for confinement in this case dynamically generate a larger effective mass for the constituent quark in the $\rho$. For the current analysis, I proceed with this assumption, taking the effective quark masses in the $\rho$ to be a conservative value, $m=m_{s}=m^{\text {eff }} \equiv 0.6 \mathrm{GeV}$.

With the mass parameters of the LF model thus fixed, and the overall normalization $N_{M}$ determined to guarantee Eq. (7), it remains to set the shape parameters $\Lambda_{M}$ that enter the relativistic vertex function in Eq. (8). To place the exact LF model in concordance with extant lattice information on the $\pi$ and $\rho$ structure functions, I tune the cutoff parameters such that the first moment of the mesons' valence distributions,

$$
\langle x\rangle_{M}=\int_{0}^{1} d x x q_{M}^{\mathrm{LF}}(x),
$$

agrees with the central values predicted by lattice calculations reported for the $\pi$ in Ref. [25] and in Ref. [26] for the $\rho$. As might be expected on the basis of Eqs. (9) and (14), the distributions $q_{M}^{\mathrm{LF}}(x)$ as well as their first moments $\langle x\rangle_{M}$ depend upon $\Lambda_{M}$ in a nonlinear fashion as shown in Fig. 2(a). Performing the necessary tuning of $\Lambda_{M}$ for the $\pi$ and $\rho$, respectively, therefore leads to the rather different values

$$
\begin{aligned}
& \langle x\rangle_{\pi}=0.214(15)\left(\begin{array}{c}
+12 \\
-9
\end{array}\right) \longrightarrow \Lambda_{\pi}=0.116 \mathrm{GeV} \\
& \langle x\rangle_{\rho}=0.334(21) \longrightarrow \Lambda_{\rho}=0.550 \mathrm{GeV}
\end{aligned}
$$

which correspond to the vertical bars of Fig. 2(a). Setting the UV cutoffs according to the central values in Eq. (25) and using the mass parameter choices mentioned above fully determines the exact LF distributions $q_{\pi, \rho}^{\mathrm{LF}}(x)$ of Eqs. (9) and (14), which I plot in Fig. 2(b). For comparison, the LF distributions of the $\pi$ and $\rho$ meson are plotted alongside the GRV extraction [30] at two lower scales, $Q^{2}=0.25,5 \mathrm{GeV}^{2}$, of the $\pi$ valence quark distribution from the global analysis of the E615 Drell-Yan data [29]. This comparison illustrates that a minimal model with few degrees of freedom is capable of falling within the range of purely data-driven determinations.
With the exact LF model for the $\pi$ and $\rho$ valence distributions thus in place, the finite- $p_{z}$ corrections to the LaMET quasidistributions - the main result of this analysis - may be computed directly. Doing so involves evaluating Eqs. (21) and (23) of Sec. II with the same parameters detailed above for the exact LF calculation, keeping the overall normalization $N_{M}$ fixed as well; I choose several finite values of $p_{z}$ at which to compute, taking $p_{z}=\{1,2,3 \mathrm{GeV}\}$ as representative choices for the hadronic boost that roughly corresponds to the intermediate range of previous calculations [17] chosen as being accessible to lattice computations. This procedure allows the direct numerical verification of the relation

$$
\lim _{p_{z} \rightarrow \infty} \tilde{q}_{M}\left(x, p_{z}\right)=q_{M}^{\mathrm{LF}}(x),
$$

which represents the fundamental result of LaMET applied to mesonic structure. It is possible to make this verification analytically by considering the behavior of Eqs. (21) and (23) in the limit of infinite momentum $p_{z}$, but the detailed breaking of the equivalence in Eq. (26) at finite $p_{z}$ is highly nontrivial, and it is for such lower hadronic momenta that lattice calculations are carried out in practice.

To make this behavior explicit, I plot the $\pi$ quasidistributions $x \tilde{q}_{\pi}\left(x, p_{z}\right)$ (heavy black curves) next to their exact LF counterpart $x q_{\pi}^{\mathrm{LF}}(x)$ (lighter brown line) in Fig. 3(a), which shows the $\pi$ valence quasi-PDFs steadily approaching the $\mathrm{LF}$ result as $p_{z}$ is increased from $1 \mathrm{GeV}$ (dotted line), to $2 \mathrm{GeV}$ (dashed), to $3 \mathrm{GeV}$ (solid), at which the point the $x$ dependence of the quasi-PDF very closely mirrors that of the exact distribution-deviating significantly only for $x \gtrsim 0.8$. To gauge the numerical size of these finite- $p_{z}$ corrections in $\tilde{q}\left(x, p_{z}\right)$, I find it useful to construct and plot a discrepancy parameter, which I define as

$$
\delta_{M}\left(x, p_{z}\right) \equiv \frac{\tilde{q}_{M}\left(x, p_{z}\right)}{q_{M}^{\mathrm{LF}}(x)}-1 ;
$$

this object quantifies as a fractional percentage the size and direction of the deviation of the LaMET meson quasiPDF from the exact LF result. For the $\pi$ distributions, I therefore pair Fig. 3(a) with a plot of $\delta_{\pi}\left(x, p_{z}\right)$ for the same collection of $p_{z}$ values in Fig. 3(b), keeping also the same correspondence between line styles and $p_{z}$. A particularly remarkable feature of these plots is the fact that $x \tilde{q}_{\pi}\left(x, p_{z}\right)$ provides a reasonable approximation to $x q_{\pi}^{\mathrm{LF}}(x)$ for $x \lesssim 0.5$ at even the smallest $p_{z}=1 \mathrm{GeV}$. The nonlinearity of the $p_{z}$ corrections - consistent with the fact that they can appear as subleading effects $\sim 1 / p_{z}^{2}$ in the formal definition of the matching for the quasi-PDF in Eq. (3) - is also strongly apparent in Fig. 3; the pronounced gap between the size of $\delta_{\pi}\left(x, p_{z}=1 \mathrm{GeV}\right)$ and $\delta_{\pi}\left(x, p_{z}=2 \mathrm{GeV}\right)$ in Fig. 3(b) suggests that incremental improvements in the reach in $p_{z}$ for lattice calculations of $\tilde{q}_{\pi}\left(x, p_{z}\right)$ would yield sharply increasing returns in affording precise access to the high 

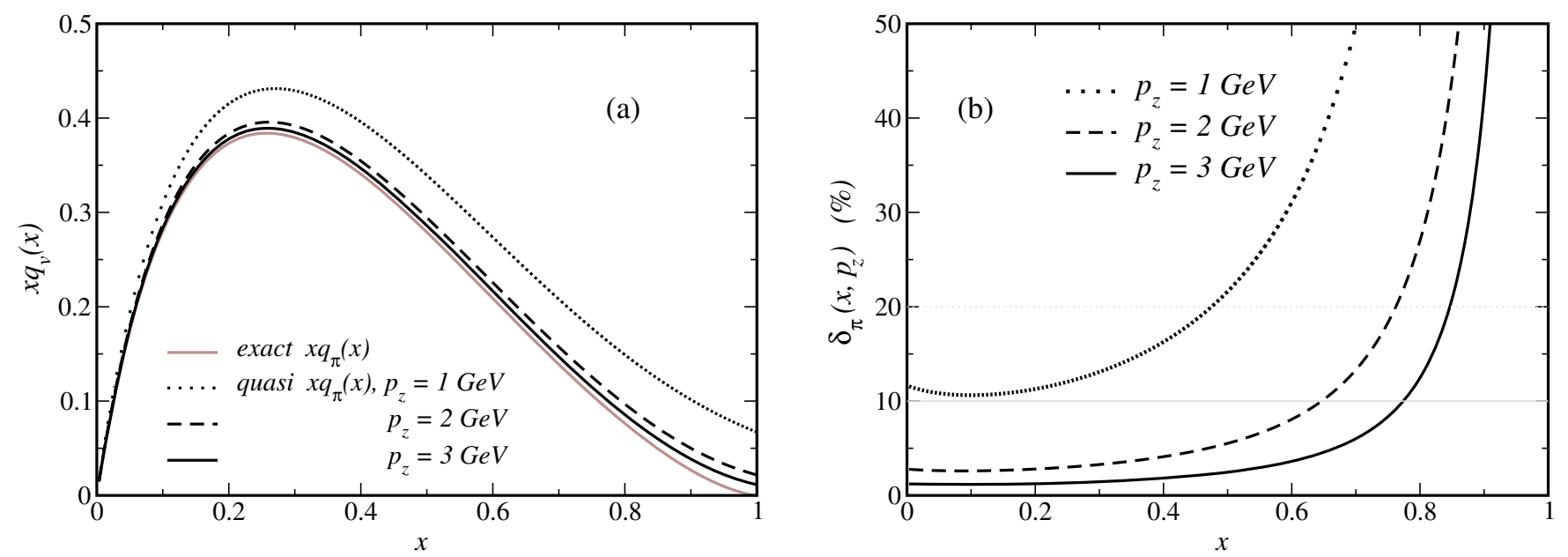

FIG. 3. (a) A plot of the exact $\pi$ distribution function $q_{\pi}^{\mathrm{LF}}(x)$ (lighter brown, solid) alongside the LaMET quasi-PDF $\tilde{q}_{\pi}\left(x, p_{z}\right)$ (heavy black curves) for several choices of $p_{z}=1 \mathrm{GeV}$ (dotted), $2 \mathrm{GeV}$ (dashed), and $3 \mathrm{GeV}$ (solid). In the limit of arbitrarily large boosts, $p_{z} \rightarrow \infty$, the black curves match onto the brown. (b) To illustrate the $x$ dependent difference between the LaMET approximation and exact valence PDF, I plot the discrepancy parameter $\delta_{\pi}\left(x, p_{z}\right)$ defined in Eq. (27), keeping the linestyles here as they were in panel (a).

$x$ region of the $\pi$ structure function. For example, with a $p_{z}$ boost of $2 \mathrm{GeV}$, Fig. 3 suggests that the quasi-PDF approximates the exact calculation to better than $10 \%$ for $x \lesssim 0.65$, while affording $\sim 20 \%$ precision in the high $x$ region until just beyond $x=0.75$.

The analogous calculation for the $\rho$ meson quasi-PDF is given in Figs. 4(a) and 4(b), which like the panels of Fig. 3 for the $\pi$ distributions compares the finite- $p_{z}$ LaMET approximation $x \tilde{q}_{\rho}\left(x, p_{z}\right)$ of Eq. (23) to the exact result $x q_{\rho}^{\mathrm{LF}}(x)$ of Eq. (14). Here again, I plot in panel (a) the $\rho$ meson quasi-PDFs themselves next to $x q_{\rho}^{\mathrm{LF}}(x)$, while panel (b) gives the associated deviations as per $\delta_{\rho}\left(x, p_{z}\right)$. Noting that performing the calculations for the $\rho$ meson according to Eqs. (14) and (23) required the use of a large effective mass for constituent quarks, the different spin structure of the $\rho$ leads to a number of differences in the distribution shapes and behavior with respect to the $\pi$ calculation. Chief among these are the different distribution curvatures I find (in general, more positive for the $\rho$ as opposed to the negative curvature of the $x q_{\pi}(x)$ distributions in Fig. 3), and the fact that the deviations $\delta_{\rho}\left(x, p_{z}\right)$ of the $\rho$ quasi-PDFs from the exact result experience a signchange, unlike $\delta_{\pi}\left(x, p_{z}\right)$, which remains strictly positivedefinite for the parameters considered. Similarly, the finite- $p_{z}$ effect in $x \tilde{q}_{\rho}\left(x, p_{z}\right)$ for the lowest choice $p_{z}=$ $1 \mathrm{GeV}$ can potentially be of larger fractional size than that
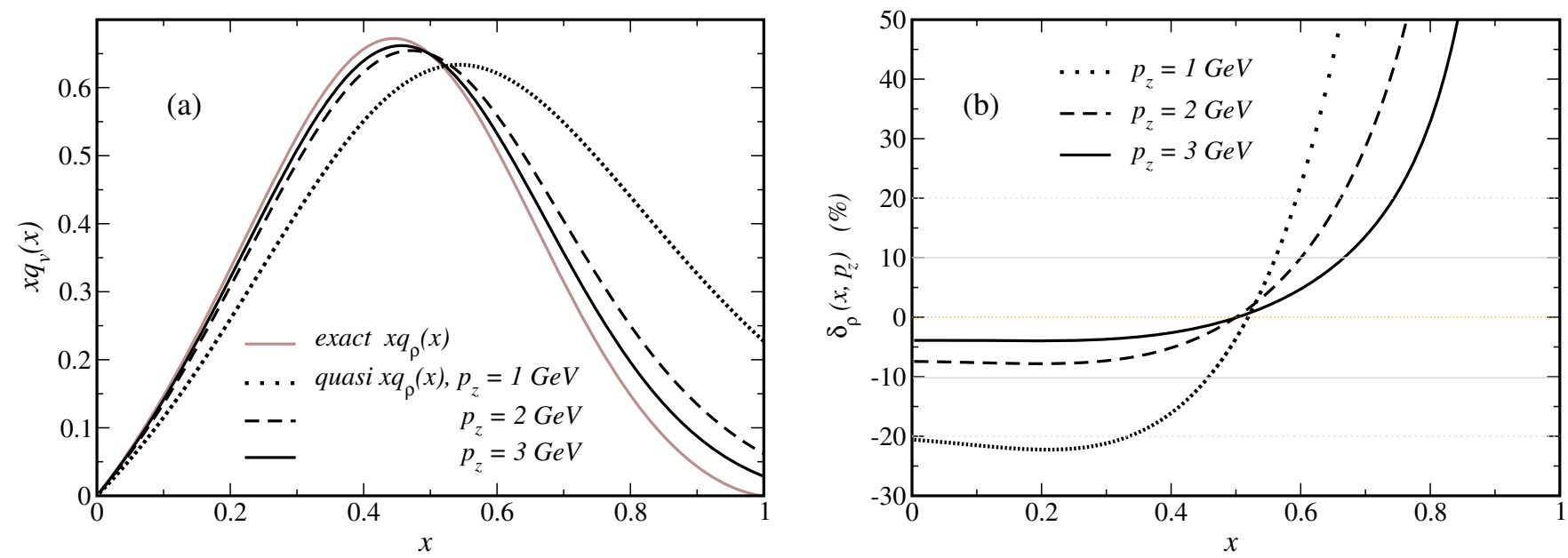

FIG. 4. Analogous to Fig. 3 for the $\pi$, panel (a) shows the exact and quasi-PDFs for the $\rho$ meson's valence structure, while (b) gives the associated discrepancy parameter $\delta_{\rho}\left(x, p_{z}\right)$. 
found for the $\pi$ distributions in Fig. 3; this is especially true at low $x$, although it should be noted that the absolute magnitude of the discrepancy remains relatively small as can be seen by comparing the curves of Fig. 4(a). As was the case for $x \tilde{q}_{\pi}\left(x, p_{z}\right)$, however, these finite- $p_{z}$ effects rapidly come under control as one moves to larger longitudinal boosts, and at $p_{z}=2 \mathrm{GeV}$ the fractional deviation is $\left|\delta_{\rho}\right| \lesssim 20 \%$ for $x \lesssim 0.65$. This suggests that lattice calculations ultimately have the potential to achieve similarly precise approximations for the $\rho$ valence distribution as for the $\pi$ via the LaMET method.

\section{CONCLUSIONS}

The foregoing represents a preliminary analysis of the collinear quark distributions of light mesons as might be achieved using the LaMET quasi-PDF formalism. Rather than focusing on the technicalities of lattice gauge theory as applied to the $\pi$ and $\rho$, I have instead restricted this study to the issue of the $p_{z}$ corrections which constitute the main limitation in the application of LaMET techniques to a given hadronic system. As 2quark systems, the $\pi$ and $\rho$ are well suited to constituent quark models such as the one presented here, suggesting that these models provide a useful avenue for assessing finite- $p_{z}$ effects.

A particular advantage of the present formulation is its simplicity: constituent quark models [33] do not explicitly incorporate gluonic degrees of freedom but rather implement phenomenological quark-meson interactions such as are given by Eqs. (4) and (12). In consequence, both LF and quasi-PDFs can be evaluated as matrix elements involving constituent quark operators according to Eqs. (1) and (2), a fact which then allows the direct numerical computation of finite- $p_{z}$ effects in the context of the model. As shown in Eq. (3), the detailed matching between the quasi-PDF and exact LF PDF is in general governed by both the perturbative UV factor $Z$ and subleading corrections $\sim M^{2} / p_{z}^{2}$. For the reasons mentioned above, however, the perturbative QCD diagrams that in general contribute to $Z$ have no direct analogue in constituent quark models like the present one; although this fact is an advantage in the sense that direct evaluation of the relevant gluon exchange diagrams is not necessary for the present calculation, an understanding of the origin of the $p_{z}$ effects described in Sec. III from perturbative QCD dynamics would be separately interesting. A more detailed investigation of this issue is reserved to a future study.
The most important conclusion of this analysis is the fact that the corrections arising from finite- $p_{z}$ effects in the $\pi$ quasidistribution $x \tilde{q}_{\pi}\left(x, p_{z}\right)$ may be potentially milder than those found in previous analyses for the nucleon quasidistribution, including Ref. [17]. By this, I mean that the size of the finite- $p_{z}$ effect in the $\pi$ quasi-PDF at high $x$, e.g., $x=0.7$, is found to be smaller in the present model calculation $\left(\delta_{\pi} \lesssim 15 \%\right.$ at $\left.p_{z}=2 \mathrm{GeV}\right)$ than for the nucleon quasi-PDF calculation of Ref. [17], which found a factor of 2-3 effect for the unpolarized $x u(x)$ distribution at $p_{z}=$ $2 \mathrm{GeV}$ using a quark-diquark model. In fact, for $x \tilde{q}_{\pi}\left(x, p_{z}\right)$ I predict that the valence PDF should be accessible to lattice calculations by means of LaMET technology to at least $20 \%$ precision for $x \lesssim 0.5$ at hadronic boosts as small as $p_{z}=1 \mathrm{GeV}$. At such a small boost, the calculation for the $\rho$ meson quasi-PDF promises to be somewhat more fraught on the other hand, with the associated $p_{z}$ corrections correspondingly larger, owing mainly to the larger $\rho$ mass; extending lattice calculations to higher boosts (e.g., $p_{z}=2 \mathrm{GeV}$ ) has the potential to ameliorate these effect, however, and the quasi-PDF discrepancies $\delta_{\rho}$ of Eq. (27) begin to resemble those of the $\pi$ at these larger momenta.

These observations augur well for the applicability of LaMET-motivated calculations of the light meson PDFs on the lattice, assuming other technical aspects for carrying out lattice gauge calculations for such light hadrons can be managed. A lattice computation of the $\pi$ valence quasi-PDF especially has the potential to hugely impact hadronic structure phenomenology and QCD global analyses [34] and should therefore be pursued as computational resources become increasingly available. This study also suggests that a future symbiosis between quark models like the present one and forthcoming lattice calculations may prove crucial to understanding the results of lattice work-including the $p_{z}$ dependence of computed quasi-distributions and extrapolations thereof to infinite momentum.

\section{ACKNOWLEDGMENTS}

I am grateful to Mary Alberg, Silas Beane, Huey-Wen Lin, Gerald Miller, Pavel Nadolsky, Fred Olness, and Xilin Zhang for enlightening discussions during the formative stages of this project. I also thank Gerald Miller for his critical reading of an early draft of the manuscript. This work was materially supported by the U.S. Department of Energy Office of Science, Office of Nuclear Physics, under Award No. DE-FG02-97ER-41014 and also by the U.S. Department of Energy under Award No. DE-SC0010129. 
[1] X. Ji, Phys. Rev. Lett. 110, 262002 (2013).

[2] A. W. Thomas, S. Theberge, and G. A. Miller, Phys. Rev. D 24, 216 (1981).

[3] J. C. Peng et al. (NuSea Collaboration), Phys. Rev. D 58, 092004 (1998).

[4] J. Annand, D. Dutta, C. Keppel, P. King, and B. Wojtsekhowski (spokespersons), Jefferson Lab Experiment PR12-15-006.

[5] A. Szczepaniak, C.-R. Ji, and S. R. Cotanch, Phys. Rev. D 49, 3466 (1994).

[6] T. Frederico and G. A. Miller, Phys. Rev. D 50, 210 (1994).

[7] W. Bentz, T. Hama, T. Matsuki, and K. Yazaki, Nucl. Phys. A651, 143 (1999).

[8] M. B. Hecht, C. D. Roberts, and S. M. Schmidt, Phys. Rev. C 63, 025213 (2001).

[9] X.-d. Ji, J.-P. Ma, and F. Yuan, Phys. Lett. B 610, 247 (2005).

[10] S. R. Beane, and T. J. Hobbs, Ann. Phys. (Amsterdam) 372, 329 (2016).

[11] J.-W. Chen, X. Ji, and J.-H. Zhang, Nucl. Phys. B915, 1 (2017).

[12] A. Radyushkin, Phys. Lett. B 767, 314 (2017).

[13] A. Radyushkin, Phys. Lett. B 770, 514 (2017).

[14] C. E. Carlson and M. Freid, Phys. Rev. D 95, 094504 (2017).

[15] H.-W. Lin, J.-W. Chen, S. D. Cohen, and X. Ji, Phys. Rev. D 91, 054510 (2015).

[16] J.-W. Chen, S. D. Cohen, X. Ji, H.-W. Lin, and J.-H. Zhang, Nucl. Phys. B911, 246 (2016).

[17] L. Gamberg, Z.-B. Kang, I. Vitev, and H. Xing, Phys. Lett. B 743, 112 (2015).
[18] A. V. Radyushkin, Phys. Rev. D 95, 056020 (2017).

[19] W. Broniowski and E. Ruiz Arriola, Phys. Lett. B 773, 385 (2017).

[20] S.-i. Nam, Mod. Phys. Lett. A 32, 1750218 (2017).

[21] J.-H. Zhang, J.-W. Chen, X. Ji, L. Jin, and H.-W. Lin, Phys. Rev. D 95, 094514 (2017).

[22] X. Xiong, X. Ji, J.-H. Zhang, and Y. Zhao, Phys. Rev. D 90, 014051 (2014).

[23] Z.-B. Kang and J.-W. Qiu, Phys. Rev. D 79, 016003 (2009).

[24] J. Collins, Foundations of Perturbative QCD (Cambridge University Press, Cambridge, England, 2013).

[25] A. Abdel-Rehim et al., Phys. Rev. D 92, 114513 (2015); 93, 039904 (2016).

[26] C. Best, M. Gockeler, R. Horsley, E.-M. Ilgenfritz, H. Perlt, P. E. L. Rakow, A. Schafer, G. Schierholz, A. Schiller, and S. Schramm, Phys. Rev. D 56, 2743 (1997).

[27] G. R. Farrar and D. R. Jackson, Phys. Rev. Lett. 35, 1416 (1975).

[28] K. Wijesooriya, P. E. Reimer, and R. J. Holt, Phys. Rev. C 72, 065203 (2005).

[29] J. S. Conway et al., Phys. Rev. D 39, 92 (1989).

[30] M. Gluck, E. Reya, and A. Vogt, Z. Phys. C 53, 651 (1992).

[31] J. Pumplin, Phys. Rev. D 73, 114015 (2006).

[32] T. J. Hobbs, J. T. Londergan, and W. Melnitchouk, Phys. Rev. D 89, 074008 (2014).

[33] T. J. Hobbs, M. Alberg, and G. A. Miller, Phys. Rev. C 95, 035205 (2017).

[34] H.-W. Lin et al., arXiv:1711.07916. 